\title{
Instituciones, transición demográfica y riesgos del sistema de pensiones
}

\author{
EnRiQue LeONARdo Kato VidaL * \\ Claudia Cárdenas Aguilar **
}

\section{RESUMEN}

Factores como el aumento en la esperanza de vida, la precariedad laboral y la escasez de empleos para adultos mayores afectan los sistemas de pensiones. Nuestra hipótesis establece que la migración del sistema de pensiones de reparto al de capitalización individual no elimina el riesgo financiero causado por la longevidad de la población. Se sigue la estrategia de contrastación de Poterba $(2001 ; 2004)$, quien relaciona la estructura poblacional con la tasa de interés en Estados Unidos y Canadá. Se concluye que la movilidad internacional de capitales benefició a los países que envejecieron primero y que actualmente tenemos un mayor reto para lograr pensiones suficientes.

Palabras clave: Envejecimiento poblacional, tasa de interés, sistemas de pensiones, México.

\section{ABSTRACT}

Factors like a rising life expectancy, precariousness in the job market, and lack of jobs for older adults affect pension systems. Our hypothesis states that the move from the distributive system to one based on personal capitalization accounts does not eliminate the financial risks posed by the population's increasing longevity. We follow Poterba's contrast strategy (2001, 2004), which correlates population structure with the interest rate in the United States and Canada. The authors conclude that international capital mobility benefited the countries that aged first and that we are currently facing a greater challenge to achieving adequate pensions. Key words: population aging, interest rate, pension systems, Mexico.

\footnotetext{
* Profesor de tiempo completo, Facultad de Contaduría y Administración (FCA), Universidad Autónoma de Querétaro, enrileo@gmail.com

** Colaboradora del Cuerpo Académico Consolidado de Innovación y Cultura, FCA, Universidad Autónoma de Querétaro,clauzzz@gmail.com
} 


\section{INTRODUCCIÓN}

El problema al que nos enfrentamos al estudiar los sistemas de pensiones es que, en la mayoría de los países, a partir de las últimas décadas del siglo xx y lo que va del siglo xxI, se observan aumentos constantes en la esperanza de vida después de la edad de jubilación y una disminución paulatina en las tasas de natalidad, al mismo tiempo que los acuerdos informales basados en la comunidad y la familia se debilitan (World Bank, 1994). El resultado para algunos es una inminente crisis causada por el envejecimiento de la población que amenaza no sólo a los adultos mayores, sino a las futuras generaciones, las cuales tend rán que asumir, directa o indirectamente, gran parte de la carga que representa proporcionar ayuda a los ancianos. Dicha situación pone en riesgo la sustentabilidad de los sistemas de pensiones, así como las finanzas familiares. Una síntesis de esta problemática la ofrece el Fondo Monetario Internacional (IMF, 2012) al advertir que la longevidad creciente representa un riesgo para generar el ingreso suficiente y con ello mantener la dinámica económica que impone la transición demográfica.

Los sistemas de pensiones son una institución cuyo propósito es proveer a los pensionados el ingreso suficiente que les permita mantener un nivel y calidad de vida acordes al que tenían antes de la edad de retiro. Las instituciones surgen como soluciones consensuales a problemas colectivos. Son un conjunto de organizaciones públicas y privadas que funcionan como mecanismos normativos, jurídicos y socioculturales, conformadas a través de un conjunto de ideas, creencias, valores y reglas que condicionan las formas del intercambio social. La principal función de una institución en la sociedad es reducir la incertidumbre, otorgando estabilidad a las interacciones humanas (Scott, 2001). En ese sentido, los sistemas de pensiones son de suma importancia para el desarrollo de una economía, ya que son instancias que procuran normalizar el comportamiento de un grupo de individuos, tanto que trascienden las voluntades individuales al identificarse con un propósito que se considera un bien social.

Desde el enfoque económico, se pretende que la institución de los sistemas de pensiones procure el desarrollo de nuestras sociedades a través de una transición de un nivel económico concreto a uno más avanzado. Este desarrollo se logra a través de un proceso de transformación estructural del sistema económico a largo plazo, con el consiguiente aumento de los factores productivos disponibles y orientados a su mejor utilización; teniendo como resultado un crecimiento equitativo entre los sectores de la producción. El desarrollo implica mejores niveles de vida para la población y no sólo un crecimiento del producto, por lo que representa cambios cuantitativos y cualitativos. 
Cabe preguntarse ¿cómo logran los sistemas de pensiones proporcionar ingresos a la población pensionada? y ¿cómo obtener ingresos suficientes para mantener la dinámica económica cuando existe un alto porcentaje de población adulta mayor? El principal problema reside en el inesperado aumento de la longevidad, que significa un riesgo financiero para los administradores de los fondos y para los que proveen los beneficios otorgados por las pensiones, quienes tendrán que asumir los crecientes pagos derivados de la seguridad social y de las pensiones, por encima de lo pronosticado.

Al tiempo que México transita en sus primeras etapas hacia el envejecimiento poblacional, Edwin van Gameren (2010) considera que en nuestro país algunos trabajadores que actualmente contribuyen a un plan de jubilación podrían no recibir una pensión mínima cuando hayan llegado a la edad de retiro, ya que no cumplen con el requisito del número mínimo de años de contribución. La situación es aún más crítica, porque en México cerca del 60 por ciento de la población ocupada no cuenta con seguro social a través de sus empleos y sólo cerca de quince millones de personas están cubiertas, lo cual significa apenas un tercio de la población económicamente activa (PEA).

Un análisis crucial para nuestro trabajo es la relación que subyace entre el envejecimiento poblacional y su efecto sobre las tasas de interés, ya que éstas determinan en gran medida los beneficios que recibirán los pensionados, derivados de sus ahorros para la jubilación. Un indicio de la influencia del envejecimiento poblacional en las tasas de interés se halla en los trabajos de Poterba (2001), quien derivó las estimaciones anuales de la demanda agregada de activos financieros y analizó los retornos de inversión. Con información sobre la distribución por edades de la población en Estados Unidos entre 1926 y 1999, sus estimaciones reflejan el perfil de edad de la demanda de activos financieros, aunque no reflejan la demanda de activos en los planes de pensiones de cuentas individuales de capitalización con prestación definida, que si bien han disminuido en importancia, aún representan una parte sustancial de los mercados financieros. Posteriormente, calcula la relación entre el tamaño de la demanda global de inversión y la relación precio-dividendo de las acciones ordinarias. Poterba encuentra una relación amplia y estadísticamente significativa entre la demanda esperada de los activos, por un lado, y los precios del mercado de valores, por el otro. Lo que no sucede con los retornos reales de inversión (Poterba, 2004).

La metodología que se utiliza en esta investigación es exploratoria, descriptiva, explicativa y causal. El trabajo se divide en cinco apartados: en el primero se halla la introducción; en el segundo, se expone que la sustentabilidad financiera de los sistemas de pensiones se encuentra en riesgo, debido a la disminución en la tasa de natalidad y a la mayor esperanza de vida, es decir, al envejecimiento poblacional que había sido subestimado en épocas pasadas; el tercer apartado corresponde al crecimiento pobla- 
cional, en el que desarrollamos la concepción de la teoría económica respecto del paso del tiempo y de las decisiones intertemporales que deben enfrentar los individuos a lo largo de su ciclo de vida; en el cuarto apartado se diseña un esquema estratégico para las decisiones de inversión intertemporal con dos jugadores que envejecen en dos periodos distintos; finalmente, en el quinto apartado, se concluye que los sistemas de pensiones han proporcionado cada vez menores ingresos en las últimas décadas y se prevé que persista esta tendencia negativa. Por último, se ofrecen las conclusiones.

Un resultado relevante es que los países desarrollados, ya envejecidos y con altas tenencias de capital, se han beneficiado en épocas pasadas por la exportación de sus capitales a países en desarrollo, intensivos en mano de obra y con bajas tenencias de capital. Las precarias condiciones de jubilación del México envejecido en las próximas décadas muestran la necesidad de políticas públicas enfocadas en asegurar condiciones de bienestar a los pensionados, políticas precautorias más allá del aumento en la edad de jubilación. La "Encuesta de trayectorias laborales" (Consar, 2012b) reporta que la densidad de afiliación al Instituto Mexicano del Seguro Social (IMSS) fue del 38.2 por ciento, es decir, en promedio, de cada cien semanas, el trabajador sólo estuvo asegurado treinta y ocho semanas. Las recomendaciones van por distintos caminos: $a$ ) acrecentar los ingresos de los trabajadores, para aumentar así los fondos de pensión; $b$ ) incrementar las tasas de cobertura de la seguridad social, especialmente a jóvenes y adultos mayores, sector en el que es mayor la exclusión del empleo con prestaciones sociales y c) reconocer la densidad incompleta de cotización, es decir, que ante el hecho de que no todos los trabajadores lograrán pensionarse, se requieren mecanismos parciales de pensión adicionales al retiro de lo ahorrado, en los casos de la negativa de pensión.

\section{SUSTENTABILIDAD FINANCIERA DE LOS SISTEMAS DE PENSIONES}

Una manera de acercarnos al envejecimiento poblacional previsto para México en las próximas décadas es tomar la experiencia del continente europeo, donde se observa una mayor transición demográfica, y el caso de Estados Unidos, que actualmente está pensionando a los nacidos en la generación del baby boom. Estas experiencias sirven para referenciar la transición demográfica que comenzamos a experimentar en nuestro país y, a partir de ello, anticipar algunos efectos sobre los sistemas de pensiones. Para Estados Unidos, Canadá y Australia, las dos décadas posteriores a la segunda guerra mundial significaron el regreso a casa de hombres jóvenes, quienes después de periodos de servicio en la guerra iniciaron sus propias familias, provocando un 
considerable incremento en el número de nacimientos entre 1946 y 1964. Este incremento estratosférico en la tasa de natalidad es conocido como el baby boom, pero a partir de 1965 la tasa de natalidad cayó abruptamente. Sólo en Estados Unidos se registraron 76000000 de niños, y cerca de mil millones de nuevos nacimientos en el mundo.

Los vínculos entre los sistemas de pensiones y el aumento en la esperanza de vida después de la jubilación (que ahora tienen lugar en al menos veinte de los treinta y cuatro países de la Organización para la Cooperación y el Desarrollo Económicos, $\mathrm{OCDE}$ ) actúan como un mecanismo para difundir el costo de la pensión entre generaciones y ayudar al sistema de ingresos de jubilación a ser financieramente sostenible en el largo plazo. En México, la nueva fuerza laboral está obligada a unirse a la nueva financiación de pensiones de gestión privada, a través de Administradoras de Fondos para el Retiro (Afores), bajo un esquema de contribución definida (Whitehouse y Queiser, 2007). La misma OCDE se pregunta ¿por qué han elegido mayoritariamente los países vincular los niveles de prestaciones a la esperanza de vida, más que a la edad de jubilación? La respuesta es que si la gente se sigue jubilando a la misma edad que en la actualidad, entonces los beneficios disminuirán a medida que crece la esperanza de vida (OCDE, 2011). La idea es que la gente trabajará más para compensar el déficit privado.

Veamos cómo funciona el envejecimiento poblacional. Entre las variables fundamentales en nuestro análisis, tenemos la edad de jubilación, la esperanza de vida y la promesa de pensión. La figura 1 muestra que en los países de la OCDE, entre 1960 y el cambio de siglo, la esperanza de vida después de la edad de jubilación ha aumentado cuatro y seis años en promedio para hombres y mujeres, respectivamente, al tiempo que se espera que hacia 2050 aumente 3 y 2.4 años más para hombres y mujeres, respectivamente; aun cuando muchos países de la OCDE ya han legislado aumentos graduales en la edad de jubilación en el futuro. Al mismo tiempo, en Europa y Estados Unidos ha disminuido la tasa de natalidad.

En México, la tasa de natalidad (nacidos vivos por cada mil habitantes) ha caído drásticamente del 28.8 al 17.8 por ciento de 1990 a 2010 y sigue disminuyendo, de manera que hacia 2050 se pronostica una tasa bruta de natalidad del 11.1 por ciento, según estadísticas y pronósticos del Conapo (2012). Lo que se prevé es un envejecimiento poblacional paulatino y, posteriormente, acelerado, ocasionado por el aumento en la esperanza de vida después de la jubilación, al mismo tiempo que disminuye la tasa de natalidad. El envejecimiento poblacional, medido como el porcentaje de población activa con 65 años de edad, es del 23 por ciento en los países miembros de la OCDE, lo que los ubica en etapas avanzadas de envejecimiento, a diferencia de México (el 11 por ciento), que aún se halla en una etapa más temprana, en la que este fenómeno, dentro del proceso de transición demográfica, se ha denominado bono demográfico. 
Este hecho es similar en otros países de América Latina, como Brasil y Colombia (Van Gameren, 2010).

\section{Figura 1}

TRANSICIÓN DEMOGRÁFICA EN EUROPA, ESTADOS UNIDOS Y MÉXICO
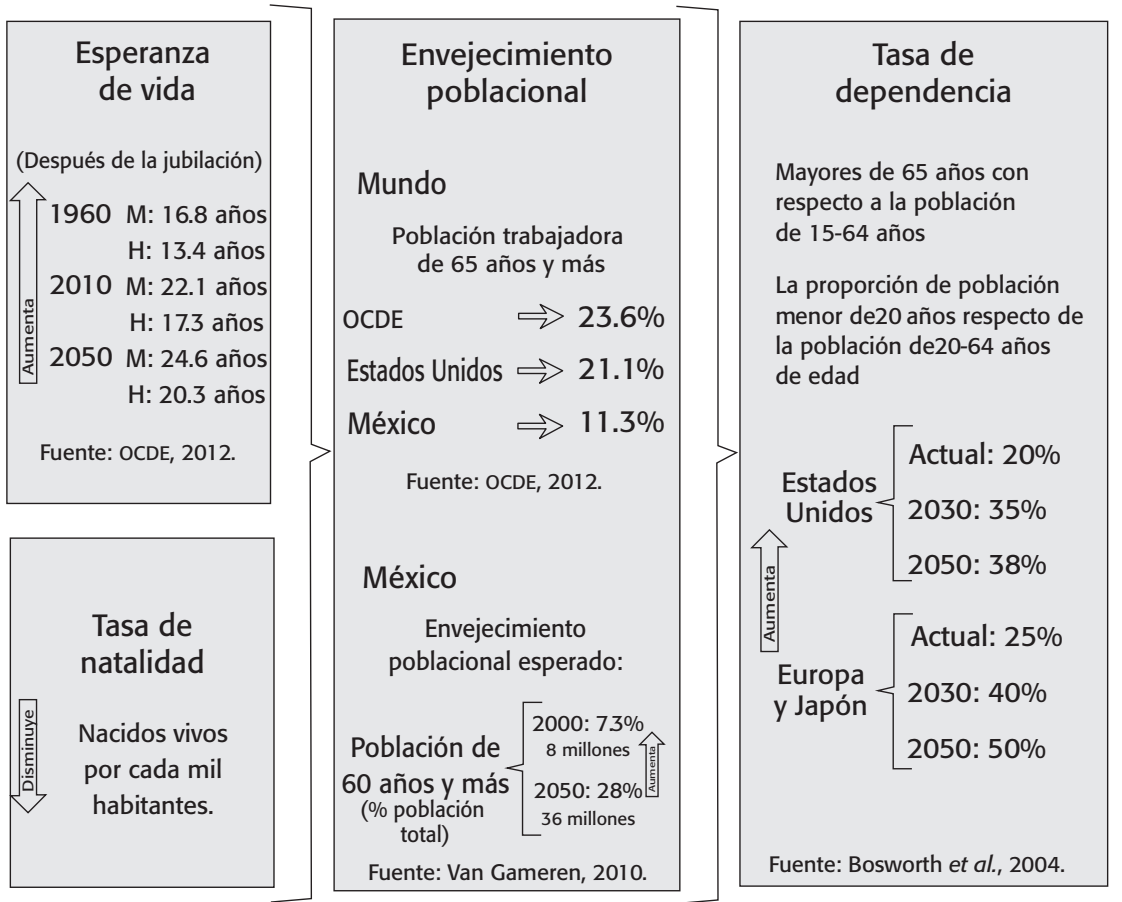

Nota: El aumento paulatino en la esperanza de vida, aunado a una disminución en la tasa de natalidad se traduce en envejecimiento poblacional acelerado.

Fuente: Elaboración propia.

El declive de las cohortes más jóvenes implica que, a mediano y largo plazo, la proporción de la gente mayor aumentará severamente en muchos países de América Latina. En México, la población mayor de 65 años de edad se encuentra estable con casi el 5 por ciento de la población total, mientras que en la población menor de 15 años de edad se ha observado una disminución del porcentaje de población alrededor del 30 por ciento, el punto en el que otros países se encontraban en 1970. Se espera que la población de edad avanzada en México comience a crecer de forma similar a la de otros países (Van Gameren, 2010), donde la población de 60 años y más crezca de entre ocho millones, en el año 2000, a más de treinta y seis millones, en 2050, 
incrementándose del 7.3 por ciento existente en el año 2000 al 17.5 en 2030 y al 28 por ciento en 2050 .

La tasa de dependencia de la población envejecida se expresa como el número de personas en edad laboral por cada persona en edad dependiente. En los países donde la tasa de dependencia de la población envejecida va en aumento, cada vez hay más personas con derecho a solicitar un plan de pensión (más adultos mayores) y se espera que descienda el número de individuos que contribuyen a financiar los planes de pensiones, debido a la disminución de la fuerza de trabajo, lo que a su vez reduce las posibilidades para obtener una pensión. Según van Gameren (2010), en la actualidad la situación de los ingresos de los adultos mayores no es buena, en parte por la falta de una pensión de retiro, ya que, para los trabajadores, las consideraciones financieras son importantes en el momento de tomar la decisión de retirarse o continuar trabajando.

En relación con los planes de retiro, poco más del 60 por ciento de la PEA no cuenta con seguro social a través de sus empleos y apenas un tercio de los trabajadores se encuentra cubierto: cerca de quince millones de personas. Aunque la seguridad social no implica precisamente participar en un programa de pensión, ésta serviría como indicador, pues van Gameren (2010) considera que algunos trabajadores que actualmente contribuyen a un plan de pensión quizá no recibirán una pensión mínima una vez que hayan llegado a la edad de retiro, al no cumplir con la regla del número mínimo de años de contribución. Para los nuevos participantes del mercado de trabajo, el ingreso en la vejez no está garantizado. Las generaciones de trabajadores que se jubilen bajo el nuevo esquema no sabrán lo que implica una promesa de pensión, ya que este esquema ha desaparecido para quienes no logren los requisitos mínimos. Según cálculos actuariales realizados por diversos investigadores, no lograr una pensión en el futuro no solamente dependerá de cumplir el requisito del tiempo cotizado, pues aun cuando se cumpla con éste, queda pendiente saber el valor de los recursos acumulados, de ello dependerá no sólo la negativa de pensión, sino el acceso a una pensión mínima, o bien a retirar los recursos en una sola exhibición, sin más beneficios. ${ }^{1}$

La participación laboral y la obtención de una pensión de retiro son algunas opciones para los individuos, pero no son todas, ya que las personas pueden decidir seguir trabajando aún después de recibir una pensión al final de su carrera laboral en un empleo o no. Posterior a la reforma a la Ley del ISSSTE de 2007, si una persona se jubila ya no podrá emplearse nuevamente en un trabajo formal, pues tendría que renunciar a la jubilación, esto en virtud de que ahora se permite la portabilidad de derechos. Un análisis para México sobre la decisión conjunta de participación laboral y retiro

\footnotetext{
${ }^{1}$ Agradecemos a uno de los dictaminadores anónimos esta observación.
} 
considera las siguientes situaciones (Van Gameren, 2010): a) sin empleo, sin beneficios de retiro; $b$ ) sin empleo, sólo beneficios de retiro; $c$ ) trabajo informal, sin beneficios de retiro; $d$ ) trabajo formal, sin beneficios de retiro y $e$ ) beneficios de retiro en combinación con un empleo formal o informal. La muestra utilizada sólo incorpora datos de hombres, ya que la mayor proporción de las mujeres de más de cincuenta años no tiene un empleo ni recibe una pensión y la mayoría nunca se empleó en un trabajo remunerado, por lo que no contribuyó a un plan de pensión.

Si se consideran las condiciones precarias y los retos que enfrentan los sistemas de seguridad para la vejez, el análisis de 2003 sobre el empleo y las pensiones para el retiro es alarmante, al mostrar que el 47 por ciento de la población adulta mayor en México no tenía un empleo ni contaba con una pensión; a la vez que el 28.5 por ciento de la población estaba ocupada en un empleo informal y, en consecuencia, no contaba con una pensión. Esto muestra que alrededor del 75 por ciento de la población total está desprotegida del riesgo en la vejez. ¿Qué pasa con el sistema de pensiones?, ¿de qué manera se asegura el consumo futuro de las personas que deberían estar ahorrando para su edad de retiro de la actividad laboral?

\section{CRECIMIENTO POBLACIONAL Y DECISIONES INTERTEMPORALES}

La relevancia de la hipótesis del ciclo vital (HCV) para un país depende de la existencia de un núcleo suficiente de hogares que sean capaces de trasladar al futuro recursos suficientes para procurar la vejez, con un nivel de vida acorde con el de antes de la jubilación (Mankiw, 2005). En este modelo, un individuo uniforma su consumo durante su vida, ahorra y acumula riqueza durante sus años de trabajo para desahorrar su riqueza y financiar su jubilación (Modigliani, 1966). El modelo de generaciones traslapadas (Blanchard y Fischer, 1989) es utilizado frecuentemente porque permite estudiar las implicaciones agregadas del ahorro del ciclo de vida de los individuos. Dicho modelo sostiene que el stock o acervo de capital es generado por los individuos que ahorran durante sus vidas laborales para financiar su consumo durante el retiro. Parte del supuesto de que la población se renueva (recambio poblacional), lo que significa que continuamente nacen nuevos individuos que sustituyen a quienes van muriendo.

La vida para cada individuo presenta dos periodos definidos: 1) de actividad económica (trabajador activo) y 2) de jubilación (trabajador pasivo). La utilidad de un individuo depende del consumo en ambos periodos de su vida y busca siempre maximizar su utilidad sujeta a su restricción presupuestaria. El tipo de interés determina qué porcentaje de los ingresos consumirá en el primer periodo y el ahorro generado en éste determinará el consumo en la segunda etapa (Romer, 2002). Se intenta 
mostrar que la influencia de la seguridad social sobre el crecimiento económico, denotado por su efecto en el ahorro privado y el stock de capital, depende de la solidaridad intergeneracional por parte de los individuos. La seguridad social permite que cada individuo pague una contribución al sistema previsional cuando es joven, en el primer periodo, para que cada jubilado reciba una pensión cuando es mayor, en el segundo periodo (López, 2006).

En el sistema de capitalización (Fully Funded System), la aportación es definida y los beneficios de la pensión están en función del rendimiento de las contribuciones que el individuo hizo en el tiempo, donde $r$ es la tasa de rentabilidad del sistema. Por su parte, en el sistema de reparto (Pay-as-You-Go System) o sistema de pagos con cargos a los ingresos corrientes, los beneficios son definidos y dependen de las contribuciones de los individuos jóvenes, bajo el supuesto de que el pensionado ya aportó en su juventud para el pago de las pensiones de los jubilados, donde $n$ es la tasa de rentabilidad del sistema que depende del crecimiento poblacional (López, 2006). Específicamente en los sistemas de capitalización individual, la seguridad social proporciona una tasa de rentabilidad igual a la del ahorro privado, siendo el consumo indiferente al tipo de ahorro realizado por el individuo.

Por ello no existen efectos del sistema de capitalización sobre el ahorro total y sobre la acumulación. En nuestra discusión, esto significa que el comportamiento optimizador del sujeto se traduce en la conocida relación marginal de sustitución entre consumos: el ahorro privado o público, en este caso no altera el nivel de utilidad del sujeto. Respecto del sistema de reparto, podemos afirmar que la tasa de rentabilidad del ahorro en seguridad social es la tasa de crecimiento poblacional, en vez de la tasa de interés, debido a que las pensiones de los jubilados en el periodo en que reciben su pensión se financian mediante las cotizaciones de los trabajadores existentes en ese instante. El equilibrio presupuestario del sistema de seguridad social exige que el total de las contribuciones hechas por la cantidad de individuos que trabajan en un mismo periodo, sea igual a las contribuciones hechas por la cantidad de individuos que trabajarán en el siguiente periodo. Por lo que la tasa de rentabilidad del sistema es la tasa de crecimiento de la población. El gobierno puede pagar una rentabilidad $n$ porque en cada periodo cada vez hay más individuos que contribuyen a la seguridad social, suponiendo igualdad de ingresos. Debido a que éste es un sistema de transferencias, y no de ahorro, la única fuente de capital de la economía es el ahorro privado.

Por definición $\mathrm{S}=\mathrm{Y}-\mathrm{C}$, el ahorro $(\mathrm{S})$ depende del ingreso $(\mathrm{Y})$ una vez deducido el consumo (C). Para estudiar la realidad de México aceptamos el supuesto de no ponzi $(Y \geq C)$ que es una condición de solvencia (Kamihigashi, 2006). Cuando se formula el problema del consumidor, este supuesto es una restricción sobre la deuda. Esta condición obliga a que el valor presente de la riqueza en el infinito no sea nega- 
tivo. Bajo estas consideraciones, podríamos diferenciar entre los participantes del sistema de pensiones y los excluidos:

- Caso 1. La renta actual es igual al consumo actual $(Y=C)$, lo que implica que el ahorro es nulo, podemos afirmar que quienes se encuentren en esta situación están excluidos del sistema de pensiones, al no incurrir en ahorro voluntario ni obligatorio. Para los trabajadores que se encuentren bajo esta condición, el consumo está condicionado al ingreso laboral obtenido en cada periodo.

- Caso 2. La renta es mayor que el consumo ( $\mathrm{Y}>\mathrm{C})$, el consumo del segundo periodo depende de $(1+r) S$, es decir, de la tasa de interés y del ahorro. Los trabajadores considerados en esta situación financian su consumo futuro a partir de dos fuentes: $a$ ) su ingreso laboral y $b$ ) su ingreso financiero, que depende del ahorro y la tasa de interés.

De acuerdo con la teoría neoclásica del crecimiento, el lento crecimiento de la fuerza de trabajo asociada con el envejecimiento poblacional debe reducir la demanda de inversión interna, compensada en parte o totalmente por la disminución esperada en el ahorro nacional. Una cuestión fundamental para los futuros retornos de inversión y para los flujos transfronterizos de capital es si el envejecimiento poblacional en los países de altos ingresos reducirá la tasa de inversión interna más de lo que se reduce el ahorro. Para los países industrializados de altos ingresos, los cuales están envejeciendo más rápido que los países en desarrollo, la demanda interna de fondos de inversión podría inicialmente caer más rápido que el ahorro nacional, causando con ello que los fondos de inversión se dirijan a países en desarrollo, donde existe una fuerte demanda de inversión. Faltaría explicar por qué para los países industrializados en su conjunto es viable mantener grandes y sostenidos excedentes de cuenta corriente con las naciones en desarrollo, lo que equivale a la exportación de una parte de sus altos ahorros para financiar inversiones productivas en estos países.

Conviene precisar que el fenómeno de financiarización, la búsqueda de rendimientos máximos, al tiempo que se subestiman los riesgos, ha eliminado gradualmente la separación entre los mercados de dinero de corto plazo y el de capitales de largo plazo, mediante la utilización de opciones financieras, futuros títulos de deuda, etc. (Correa et al., 2013: 259-260). Los fondos de pensión o inversionistas institucionales han aportado enormes cantidades de dinero al circuito financiero para cubrir sus compromisos. Así, aunque los fondos sean de los trabajadores de una nación, estos recursos pueden financiar actividades en cualquier parte del mundo. Para países como México, la financiarización ha significado mayor presión sobre la actividad productiva para la obtención de ganancias. 
En términos económicos, se clasificaría a las naciones en desarrollo como intensivas en trabajo, calificativo que surge de su abundancia relativa de mano de obra. En contraparte, las naciones desarrolladas se califican como intensivas en capital, debido a la escasez relativa de trabajo y la abundancia relativa de ahorros y de bienes de capital (Dollar, 1986). La distribución desigual de factores en las naciones del mundo provoca flujos internacionales de recursos. La dirección de esos flujos es precisa. Los migrantes se dirigen a las naciones desarrolladas porque, ante la escasez de mano de obra, podrían recibir mayores salarios por su trabajo, comparados con los salarios de sus lugares de origen (Krugman y Obstreld, 2006). A su vez, los capitales que son relativamente abundantes en las naciones desarrolladas quizás reciban mayores rendimientos si se invierten en las naciones en desarrollo, como es el caso de los administradores de los fondos de pensiones. Este resultado es conocido en los modelos de economía internacional. Se basa en el principio de la productividad marginal del capital que refleja, aproximadamente, la tasa de interés o la de rendimiento sobre aquél (Caselli y Feyrer, 2005). Existe una relación inversa entre la tasa de rendimiento y la productividad del capital. Empíricamente, Asiedu (2002) utiliza el recíproco del PIB per cápita para determinar la escasez del capital en una economía. Esta aproximación permite diferenciar entre las economías exportadoras y receptoras netas de capital.

\section{INVERSIÓN, PENSIONES Y TASA DE INTERÉS}

En México, como en otros países, la población está incrementando su esperanza de vida sin aumentar simultáneamente los ahorros para la pensión. El Fondo Monetario Internacional alertó al mundo sobre un riesgo de longevidad, surgido de un aumento continuo de la esperanza de vida posterior a la jubilación. Aunque en el corto plazo sean pequeños los errores de los pronósticos de longevidad, en el largo plazo los efectos se acumulan para las finanzas públicas y privadas, y llegan a representar varios puntos porcentuales del producto nacional (IMF, 2012). La evidencia presentada por el Fondo Monetario contrasta con las tendencias en México, donde la población joven cuenta con condiciones laborales precarias en comparación con generaciones previas. Además, los sueldos actuales han perdido poder de compra y menos jóvenes cuentan con afiliación a la seguridad social. Por lo que en el IMss aumenta la edad de la población de los trabajadores derechohabientes. La Dirección de Incorporación y Recaudación del IMSS reporta que, en el 2000, seis de cada diez afiliados tenía 34 años de edad o menos, tres de cada diez pertenecía al grupo de 35 a 49 años y una de cada diez de 50 o más; en 2011, las proporciones eran de cinco, cuatro y uno de cada diez, respectivamente, para las edades de 34 años y menos, de 35 a 49 y de 50 y más. 
A finales de 2012, la Consar fusionó las Sociedades de Inversión Especializadas en Fondos para el Retiro (Siefores) SB4 y SB5. La explicación dada por la autoridad es que los trabajadores más jóvenes comienzan a trabajar en empleos informales al inicio de su vida laboral y se integran al sistema formal después de los 26 años; esto genera una afiliación directa a otras sociedades de inversión para personas de mayor edad, sin pasar por la SB5 (Consar, 2012b). Esta decisión de la Consar es consistente con el fenómeno de envejecimiento de la población afiliada en el IMSS.

La combinación de factores es muy adversa para la generación de nuevos trabajadores: bajas tasas de interés, baja cobertura a la seguridad social y bajos salarios. Debido al entorno económico de los años recientes, parece poco probable que se logren pensiones suficientes para financiar la etapa de retiro, especialmente en las nuevas generaciones. La figura 2 muestra la acumulación real de activos netos, según las Siefores básicas (SB) en el periodo 2008-2012. Los ahorros de los individuos de mayor edad se administran en las Siefores básicas SB1+SB2, los de mediana edad en la SB3 y los activos para los individuos más jóvenes en las SB4+SB5. Con la información de las Siefores se revela una inequidad intergeneracional en la que convergen dos aspectos: 1) las deterioradas condiciones laborales para los jóvenes y 2) las bajas tasas de interés prevalecientes en el mundo, como consecuencia de la crisis mundial iniciada en 2008 (la Gran Recesión). Resulta grave la combinación de ambas tendencias, porque en México la edad promedio en la que un individuo asalariado, catalogado como trabajador subordinado, obtiene los ingresos más altos de su ciclo de vida es a los 37 años. A partir de esa edad el ingreso comenzará a decrecer (ver figura 3A).

El balance se alcanza cuando existe una transición de trabajador subordinado a trabajador independiente, entre los 30 y 40 años de edad, una vez que se haya obtenido experiencia laboral y también en la búsqueda de mayores ingresos. Existe evidencia de que al principio este cambio se da en condiciones de informalidad; conforme aumenta la edad del emprendedor, también aumenta la tasa de formalidad. A pesar de ello, en los últimos años ha disminuido la propensión de los trabajadores independientes a formalizar su actividad, lo que se muestra en la figura 3B como un corrimiento hacia abajo de las curvas de proporción de trabajadores formales. De ser exitosa la transición, el trabajador logrará evitar el declive de su ingreso y podría tener mejores perspectivas para la etapa de su jubilación. El monto de su pensión dependerá de la cantidad que ahorre y de la tasa de rendimiento que obtenga.

Con el propósito de conocer la evolución de las tasas de interés y, con ello, el consumo de los pensionados, se presentan los rendimientos en una perspectiva de largo plazo. Analicemos el periodo de 1955 a 2011 de distintos instrumentos financieros de Estados Unidos, que es el punto de referencia para muchas economías del mundo (ver figura 4). De hecho, es una práctica usual en los mercados internaciona- 
Figura 2

ACUMULACIÓN REAL DE ACTIVOS NETOS, SEGÚN SIEFORES BÁSICAS (NÚMERO ÍNDICE DEL PROMEDIO MÓVIL 3)
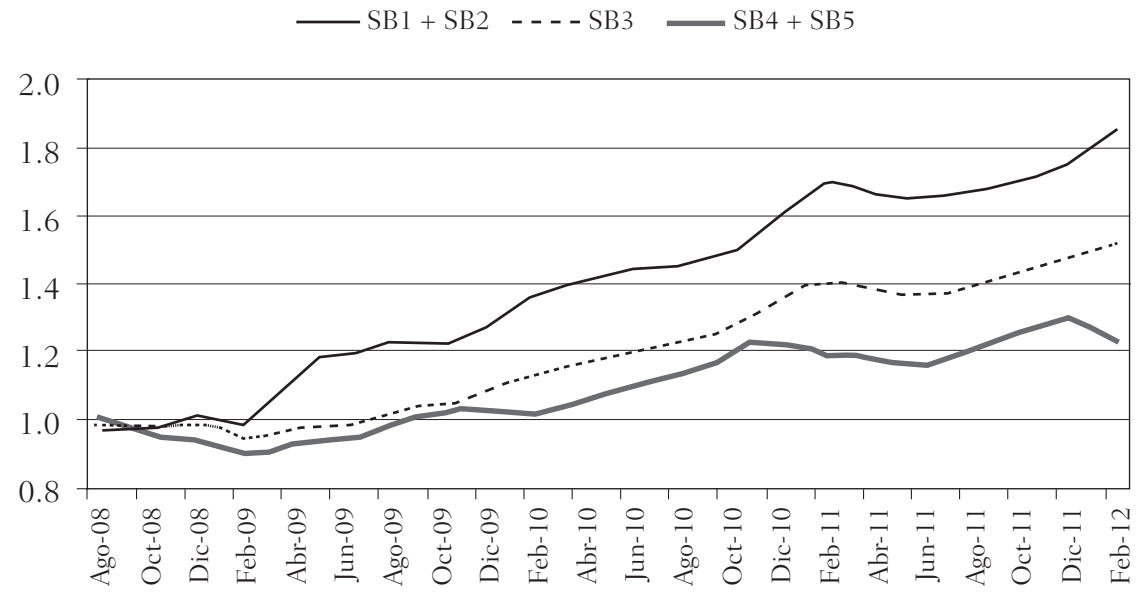

Nota: SB corresponde a la Siefore básica. El índice de precios utiliza como periodo base la segunda quincena de diciembre de 2010.

Fuente: Elaboración propia, con datos de <www.consar.gob.mx〉, consultada en marzo de 2012.

les calcular el diferencial de tasas del rendimiento promedio de los instrumentos de un país con los instrumentos considerados libres de riesgo, usualmente los emitidos por Estados Unidos. Por tal motivo, podemos afirmar que las tasas de interés en México serán más altas si aumentan las tasas en Estados Unidos, o si aumenta el riesgo en el país de México. En la figura 4 se aprecia una tendencia ascendente en las tasas de interés entre 1955 y el fin de siglo. Particularmente, se registraron altas tasas de interés en el último quindecenio, que contrasta con la abrupta caída de las tasa de interés al inicio del nuevo siglo.

Respecto de los planes de pensiones, identificamos tres trayectorias a partir de las tasas de interés (ver figura 5). Para ejemplificar, se utilizan como referencia las tasas de préstamos bancarios en Estados Unidos (segundo grupo de barras de la figura 4) y se asume que la vida laboral de una persona dura aproximadamente treinta años. Se busca conocer cuál ha sido la tasa de interés y el ingreso financiero vigente durante sus años laborales. Como se ilustra en la figura 5, la primera trayectoria la asociamos con la población trabajadora de la segunda mitad del siglo xx. Las tasas de interés de los periodos 1955-1970 y 1971-1984 fueron del 2.4 y 3.1 por ciento, respectivamente ( 2.75 por ciento, en promedio simple). 


\section{Figura 3}

TRANSICIÓN DE EMPLEADO SUBORDINADO A TRABAJADOR INDEPENDIENTE, CERCA DE LOS 40 AÑOS DE EDAD

A

A los 37 años de edad el trabajador asalariado alcanza su salario máximo

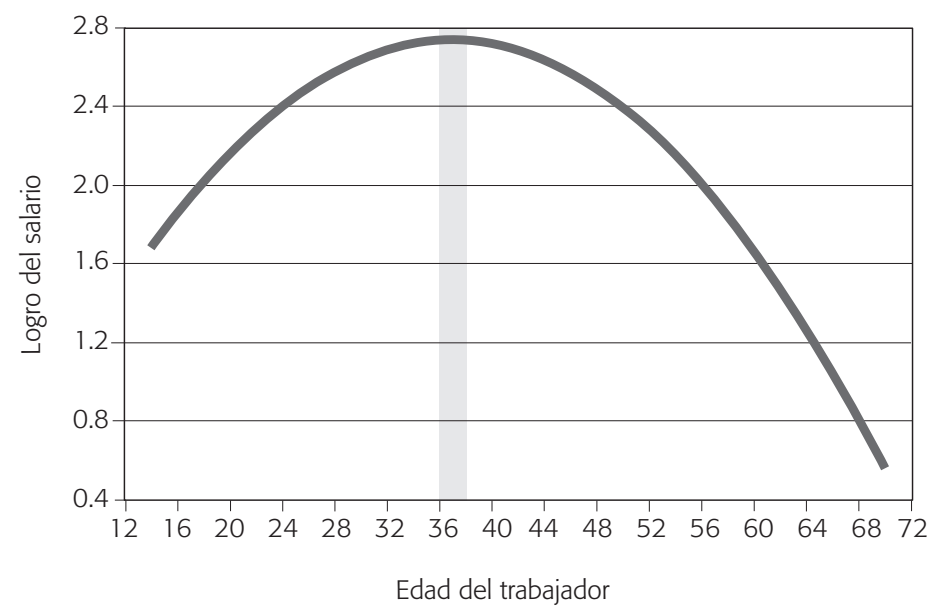

Fuente: Cálculos propios con base en las estimaciones del cuadro 3 de Valdivia López y Pedrero (2011).

B A partir de los 40 años aumenta la proporción de trabajadores independientes en el sector formal

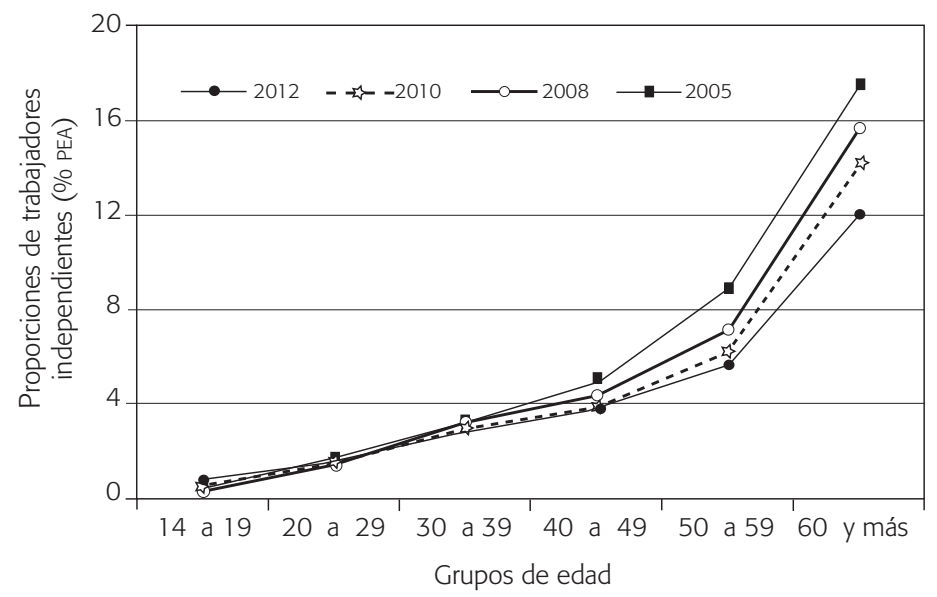

Fuente: Encuesta Nacional de Ocupación y Empleo, INEGI (varios años). 
Si comparamos este resultado con la generación que inició su etapa laboral durante los años setenta o la primera mitad de los ochenta, su tasa promedio rondará el 4.2 por ciento, ya sea que este promedio se obtenga iniciando con la tasa baja del 3.1 por ciento y terminando con un 5.2 o iniciando con un 5.2 por ciento y concluyendo con un 3.3. Lo fundamental en este planteamiento es lo que ocurrirá en la tercera trayectoria, con las personas que inician su actividad laboral a inicios del milenio. En la economía mundial ya no se registran elevadas tasas de interés como ocurrió en los años noventa. La crisis de 2008-2009 y su secuela han tenido como consecuencia bajas tasas de interés para intentar promover una senda de crecimiento. Durante los meses en que se escribió este trabajo, la crisis europea se acentuó, lo que previsiblemente tendrá como consecuencia que permanezcan más tiempo las bajas tasas de interés mundiales.

A diferencia de quienes se pensionaron en el pasado con bajas tasas, lo que hemos llamado primera trayectoria (1955-1985), la situación actual es más grave porque, desde entonces hasta la fecha, los fondos de pensión se modificaron de sistemas de reparto a fondos de capitalización individual. Lo anterior implica que aunque antes (trayectoria 1) prevalecieron los intereses bajos, éstos pudieron compensarse con altas tasas de natalidad, debido a un mayor número de contribuyentes. El cambio de régimen y la demografía han cancelado esta posibilidad y los fondos de pensiones únicamente pueden financiarse con ahorro e intereses de mercado.

La cuestión que se modelará es que el envejecimiento de la población del mundo favorece a los países que envejecen primero, toda vez que pueden obtener beneficios invirtiendo en los países con población joven. En cambio, las sociedades que envejecen tardíamente no dispondrán de los mismos beneficios, en un mundo con un alto porcentaje de población ya envejecida, sino de beneficios menores. Al utilizar la teoría de juegos, sabemos que los pagos para cada jugador dependen de su propia elección, pero también del otro jugador, por lo que el diseño de estrategias es fundamental (Dixit et al., 2009). La situación se representa en un juego secuencial en dos tiempos. En $t=1$, elige el administrador de fondos de la nación que envejece primero, es decir, de los países desarrollados (DC). Sus alternativas son invertir en DC o en los países en desarrollo (LDC). En $t=2$, el administrador de LDC se enfrenta a las mismas alternativas de inversión. Como es usual (Gibbons, 1992), los pagos $\pi$ de cada jugador dependen de su propia elección $(a)$, pero también de la elección del otro jugador, es decir, $\pi_{i}\left(a_{i}, a_{-i}\right)$. 


\section{Figura 4}

TASAS DE INTERÉS REALES EN ESTADOS UNIDOS, 1955-2011

1955-1970

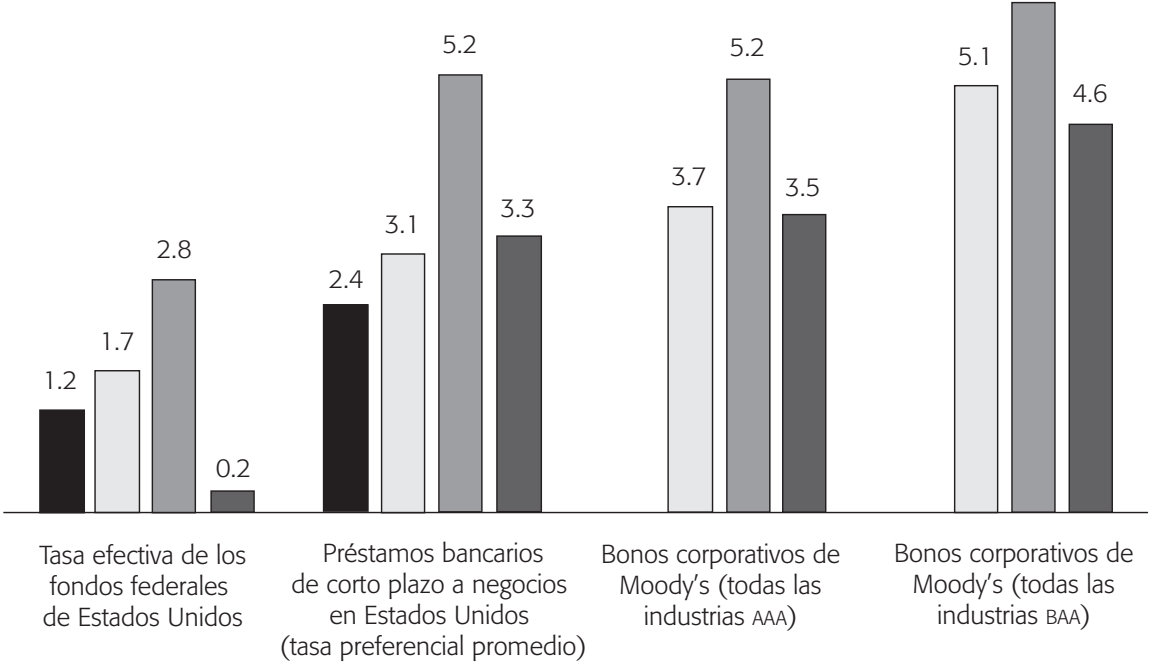

Fuente: Elaboración propia con datos de la Reserva Federal de Estados Unidos, consultada en marzo de 2012.

Figura 5

TRAYECTORIAS DE LAS TASAS DE INTERÉS RESPECTO DE LOS PLANES DE PENSIONES

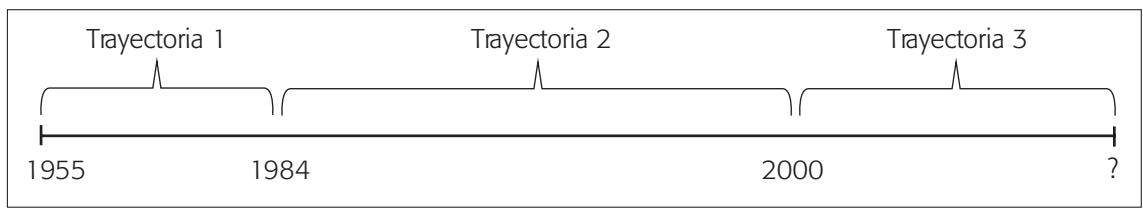

Fuente: Elaboración propia, utilizando el lapso de la figura 4.

La figura 6 muestra un juego secuencial con cuatro posibles soluciones. Los pagos en orden decreciente son $r_{6}>r_{5}>r_{4}>r_{3}>r_{2}>r_{1}$, toda vez que el producto marginal del capital presenta rendimientos decrecientes. A medida que el capital aumenta, la productividad marginal disminuye y también las tasas de interés. En la figura 6A el juego muestra una estrategia dominante de DC, la estrategia dominante es invertir en LDC, dado que le reporta un mayor beneficio que invertir en su propio país (Dixit 
et al., 2009). La solución del juego es $\left(r_{5} \mathrm{y} r_{4}\right)$, donde DC obtiene el mayor pago posible del conjunto a su alcance. Esto implica que LDC pierde la posibilidad de obtener $r_{6}$ cuando DC decide invertir en LDC, lo que se muestra eliminando la rama superior en la figura 6B. En términos estratégicos decimos que el primer jugador tiene ventaja sobre el segundo porque elige primero. Esto significa que las naciones desarrolladas (DC) usufructuan los beneficios de las naciones en desarrollo, tomando ventaja de que poseen los capitales y de que pueden disponer de la población joven en los países con bajas tenencias de capital.

\section{Figura 6}

JUEGO SECUENCIAL: VENTAJA DE LA NACIÓN QUE ENVEJECE PRIMERO

A

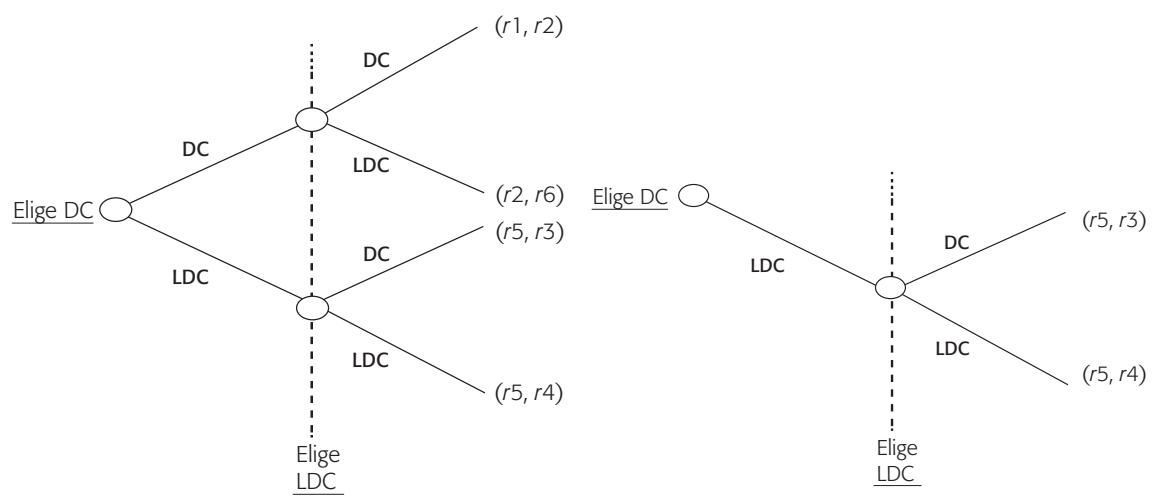

Nota: Los pagos se determinaron utilizando cuatro curvas de productividad marginal; dos para DC y dos para LDC. Las dos curvas para cada región corresponden a las dos fechas: $t=1$ y $t=2$.

Fuente: Elaboración propia.

La teoría predice que la movilidad internacional de factores permite maximizar el producto mundial. La condición de maximización establece que se exportará capital hasta que se iguale el producto marginal del capital extranjero con el nacional (Krugman y Obstfeld, 2006). Al comparar los beneficios de DC y los costos de LDC, el saldo es negativo. Esta situación se denomina teóricamente como pérdida irrecuperable de eficiencia. Los beneficios de la movilidad internacional de capital generan un costo en que incurre el país anfitrión del capital extranjero. El resultado que aquí se reporta contradice a la teoría convencional de estática comparada en economía internacional. La contradicción surge al asumir el envejecimiento de la población mundial, la cual acentúa la caída de los rendimientos decrecientes al capital. 
Un estudio que analiza la repatriación de utilidades de la inversión extranjera directa (IED) estadunidense desde México confirma que el pago de dividendos de las filiales en México a sus matrices en Estados Unidos tiene efectos positivos en las variables que miden la actividad económica en Estados Unidos; se estima que la elasticidad es mayor a uno, es decir, existe una notable contribución al nivel de vida en la vecina economía del norte (Briseño y Neme, 2011: 145). Para que la atracción de capitales se traduzca en beneficios para México, se requiere establecer una política industrial que propicie simultáneamente la inversión en capital físico y humano, junto con la capacidad e incentivos para invertir en la absorción de tecnología extranjera y en las habilidades asociadas (Briseño y Neme, 2011: 158). En tanto, únicamente se identifica que la IED contribuye a una expansión del consumo, aunque no de la inversión ni de la producción en la economía receptora de dicha inversión.

\section{Conclusiones}

El sistema de pensión de capitalización individual se adoptó presuntamente para resolver la debilidad que el envejecimiento poblacional causaba al anterior sistema de reparto. Bajo la capitalización individual, la pensión depende de la tasa de interés, que a su vez depende de la disponibilidad de población trabajadora para lograr el rendimiento que paga el sistema financiero. La transición demográfica en México es un proceso más reciente si se compara con el envejecimiento poblacional en Europa y en Estados Unidos (por ejemplo, baby boomers), pero las consecuencias económicas del envejecimiento parecen ser más altas en México, debido al bajo acceso a los sistemas de pensiones y previsiblemente a los bajos montos que se pagarán como pensión. Podemos concluir que el envejecimiento poblacional sí muestra incidencia en los rendimientos financieros a través de la influencia del crecimiento poblacional sobre la tasa de interés. Por lo que las menores tasas de interés reales de los últimos veinte años se traducirán en menores montos de pensión.

Un resultado relevante es que los países desarrollados ya envejecidos y con altas tenencias de capital se han visto beneficiados en épocas pasadas por exportar sus capitales a países en desarrollo intensivos en mano de obra y bajas tenencias de capital, lo que se traduce en una alta productividad marginal del capital y en altas tasas implícitas de rendimiento. Bajo rendimientos decrecientes, a medida que aumenta el capital disminuye su productividad, habiendo menores tasas de rendimiento. Es indispensable que quienes toman decisiones en políticas públicas reconozcan la magnitud y los impactos del envejecimiento poblacional en las finanzas públicas y privadas, para adoptar medidas más allá del aumento de la edad de jubilación. Se debe sanear no 
únicamente la institución de los sistemas de pensión, sino también las finanzas de las familias de los pensionados.

Las estimaciones muestran que a los 37 años de edad se alcanzan los ingresos máximos de los asalariados en México. A partir de esa fecha aumenta el número de trabajadores que se independiza para mantener o incrementar sus ingresos. Reconocemos que hace falta más que el esfuerzo de los trabajadores por aportar voluntariamente a sus cuentas de ahorro para el retiro, que si bien constituyen una medida precautoria importante para la jubilación, en términos agregados podría resultar insuficiente dada la baja empleabilidad, la baja cobertura y la pérdida de poder de compra de los salarios. El reto gira en torno al aumento de los ingresos de los trabajadores, ya que el acceso a las Afores es un derecho exclusivo de quienes se insertan al mercado laboral formal; el reto se complica más por la precarización de los salarios y las desventajas laborales que implican los intermediarios laborales generados por la subcontratación.

\section{Biblografía}

Asiedu, Elizabeth

2002 "On the Determinants of Foreign Direct Investment to Developing Countries: Is Africa Different?", World Development, vol. 30, no. 1, pp. 107-119.

\section{Blanchard, Oliver y Stanley Fisher}

1989 Lectures on Macroeconomic, Cambridge, MIT Press.

\section{Bosworth, B. P., R. C. Bryant y G. Burtless}

2004 "The Impact of Aging on Financial Markets and the Economy: A Survey", The Brookings Institution, Washington, D.C.

\section{BRISEÑo, José IsRael y OMAR NEME}

2011 "Repatriación de utilidades de la inversión extranjera directa: la relación entre México y Estados Unidos", Norteamérica. Revista Académica, año 6, no. 1, pp. 127-166.

Caselli, Francisco y James Feyrer

2005 “The Marginal Product of Capital”, NBER Working Paper Series, vol. 11551, s.n., pp. 3-7. 
Comisión Nacional del Sistema de Ahorro para el Retiro (Consar)

2013 "Información general de trabajadores del IMSs", en <www.consar.gob.mx/ principal/info_gral_trabajadores-imss_retiro_total_01.shtml $>$.

2012a "Adecuaciones al régimen de inversión en beneficio de los trabajadores", boletín de prensa, no. 19, 2012.

2012b "Encuesta de trayectorias laborales", México, Consar.

Consejo Nacional de Población (CONAPO)

2012 "Indicadores demográficos básicos 1990-2030", en <www.conapo.gob.mx>, consultado el 20 de marzo de 2012.

Correa, Eugenia, Gregorio Vidal y Wesley Marshall

2013 "Financialization in Mexico: Trajectory and Limits", Journal of Post Keynesian Economics, vol. 35, no. 2, pp. 255-275.

Dixit, Avinash, Susan Skeath y David Reiley

2009 Games of Strategy, Nueva York, W.W. Norton.

Dollar, DAVID

1986 "Technological Innovation, Capital Mobility, and the Product Cycle in NorthSouth Trade", American Economic Review, vol. 76, pp. 177-190.

GAMEREN, EDWIN VAN

2010 "La participación laboral de los adultos mayores", Los grandes problemas de México, México, El Colegio de México, pp. 258-306.

GIBBONS, ROBERT

1992 Un primer curso de teoría de juegos, Barcelona, Antoni Bosch.

GRUBERT, HARRY

2012 "Foreign Taxes and the Growing Share of U. S. Multinational Company Income Abroad: Profits, Not Sales, Are Being Globalized", National Tax Journal, vol. 65, no. 2, pp. 247-282.

INTERNATIONAL MONETARY Fund (IMF)

2012 "The Financial Impact of Longevity Risk", Global Financial Stability Report, Washington, D.C., IMF, pp. 1-37. 
KAMIHIGASHI, TAKASHI, ed.

2006 "Transversality Conditions and Dynamic Economic Behavior", Discussion Paper Series, Kobe, Research Institute for Economics and Business Administration, Kobe University.

Krugman, Paul y Maurice Obstfeld, eds.

2006 International Economics. Theory and Policy, Boston, Pearson.

LÓPEZ, JULio

2006 "Seguridad social y crecimiento económico", Principios: Estudios de Economía Política, no. 4, pp. 23-54

MANKIW, GREGory

2000 Macroeconomía, Barcelona, Antoni Bosch.

Modigliani, Franco

1966 "The Life Cycle Hypothesis of Saving. The Demand for Wealth and the Supply of Capital", Social Research, vol. 33, no. 2, pp. 160-217.

Organization for Economic Co-operation And Development (ocde)

2011 Pensions at a Glance 2011: Retirement-income Systems in OECD and G20, París, OECD.

POTERBA, JAMES

2004 "The Impact of Population Aging on Financial Markets", Working Paper Series, no. 10851, Cambridge, National Bureau of Economic Research.

2001 "Demographic Structure and Asset Returns", Review of Economics and Statistics, vol. 83, no. 4, pp. 565-584.

ROMER, DAVID

2002 Macroeconomía avanzada, Madrid, McGraw-Hill.

SCOTT, W. RicHARD

2001 Institutions and Organizations, Nueva York, Sage.

Valdivia, Marcos y Mercedes Pedrero

2011 "Segmentación laboral, educación y desigualdad salarial en México", Revista Mexicana de Sociología, vol. 73, no. 1, pp. 139-175. 
VARIAN, HAL

1992 Análisis microeconómico, Barcelona, Antoni Bosch.

Whitehouse, Edward y Monika QueISER

2007 Pensions at Glance: Public Policies across ocde Countries, París, OCDE.

WORLD BANK

1994 Averting the Old Age Crisis, Washington, D.C., Oxford University Press. 